MATEC Web of Conferences 11,01007 (2014)

DOI: $10.1051 /$ matecconf / 20141101007

(C) Owned by the authors, published by EDP Sciences, 2014

\title{
Influence of adjuvants on the properties of underwater cast concrete on base of cement (HRS $32.5 \mathrm{~N}$ )
}

\author{
Mohamed Jamel Rouis ${ }^{1 \text { et } 2}$, Afef Makni ${ }^{1 \text { et } 2}$ \\ ${ }^{1}$ Ecole Nationale d'Ingénieurs de Sfax, Géologie, Sfax, Tunisie \\ ${ }^{2}$ Unité de recherches Géotechnique environnementale et matériaux civils, Ecole Nationale \\ d'Ingénieurs de Sfax - Tunisie \\ e-mail1:jamel.rouis@hotmail.fr \\ e-mail22:pureau.makni@gmail.com
}

\begin{abstract}
Concrete cast in the water are a great innovation for deep concrete foundations, they are technological, economic and social. Therefore, it is important to reach a suitable formulation concrete meets the rheological criteria (flow, good wash resistance, good flow, no segregation, homogeneity, chemical compatibility) and the compressive strength. This work focuses on the study of the influence of additives on the properties of concrete applied to deep marine foundations. The experimental study aims to:

* The optimization of anti washing adjuvants and the super plasticizer.

* Follow the contribution of adjuvants for both super plasticizers and anti-washing on the rheological criteria sunk underwater concrete and grout mortars in fresh and hardened state. The laboratory tests include:

* The design of mixtures of different strengths from local materials (based on portland cement HRS 32, 5), and the optimization of adjuvant anti washout test dive to follow the role of these additives on the properties of concrete in the fresh state.

* Optimizing adjuvants super plasticizers and follow its influence on the criteria mortars fresh by the method of grout.

* The characterization tests of concrete in the hardened state including destructive and non destructive tests performed on specimens made in concrete (based on portland cement), with varying dosages and adjuvants at different times (28d and $90 \mathrm{~d})$.
\end{abstract}

Key-words: Poured concrete under water, concrete formulation, the influence of additives, properties of concrete, adjuvant anti wash, super plasticizer, cement HRS32.5, compressive strength, speed ultrasons.

Résumé. Les bétons coulés sous l'eau représentent une grande innovation pour les fondations profondes en béton, ils présentent des avantages technologiques, économiques et sociaux. De ce fait, il est important d'accéder à une formulation de béton adéquat répondant aux critères rhéologiques (fluidité, bonne résistance au lavage, bon écoulement, absence de ségrégation, homogénéité, compatibilité chimique) et à la résistance à la compression visée. Ce travail s'intéresse à l'étude de l'influence des adjuvants sur les propriétés des bétons appliqués aux fondations marines profonde. L'étude expérimentale a pour objectifs :

* L'optimisation des adjuvants d'anti lavage et du super plastifiant.

*Suivre l'apport des adjuvants aussi bien pour les super plastifiants que pour l'anti-lavage sur les critères rhéologiques des bétons coulé sous l'eau et des coulis de mortiers à l'état frais et durci. Les essais réalisés en laboratoire concernent :

* La conception de mélanges de différents dosages à partir des matériaux locaux à base de ciment portland : HRS32, 5 et l'optimisation de l'adjuvant d'anti lavage par l'essai au plongeon pour suivre le rôle de ces adjuvants sur les propriétés des bétons à l'état frais.

* Optimisation des adjuvants super plastifiants et suivre son influence sur les critères des mortiers à l'état frais par la méthode de coulis.

*Les essais de caractérisation du béton à l'état durci comprenant les essais destructifs et non destructifs effectués sur des éprouvettes confectionnées en bétons (à base de ciment portland), avec des dosages variables en adjuvants et à des dates différentes $(28 \mathrm{j}$ et $90 \mathrm{j})$.

Mots clés. Béton coulé sous l'eau, formulation du béton, influence des adjuvants, propriétés des bétons, adjuvant d'anti lavage, super plastifiant, ciment HRS 32.5, résistance à la compression, vitesse ultrasons.

\section{Introduction}

Les bétons coulés sous l'eau représentent une grande innovation pour les fondations profondes en béton, ils présentent des avantages technologiques, économiques et sociaux. Le béton coulé sous l'eau est une technique employée dans la construction d'une large gamme d'ouvrages situés en site maritime, qui contribuent à l'aménagement des infrastructures portuaires, des bassins, des bases marines, des ouvrages de protection des côtes et des ouvrages de mise à l'eau de construction nautique[1].

\section{Le béton coulé sous l'eau}

Le bétonnage sous l'eau est appliqué pour la construction de puits profonds, d'installations portuaires, de piles de ponts, ou encore lorsqu'on a affaire à une fouille sans abaissement de la nappe .On procède à des bétonnages sous l'eau aussi bien dans des eaux calmes que dans des eaux en écoulement. Le béton doit présenter

This is an Open Access article distributed under the terms of the Creative Commons Attribution License 2.0, which permits unrestricted use, distribution, and reproduction in any medium, provided the original work is properly cited. 
une bonne cohésion, ne pas être sujet au démélange et ne pas être facilement délavable, d'où la nécessité d'ajouter lors de sa fabrication un additif colloïdal. Pour réduire les pertes de béton à un minimum, on le met en place au moyen d'un tube plongeur. Ce procédé consiste à couler le béton à travers un tube de 20 à $40 \mathrm{~cm}$ de diamètre. Le tube est alors remonté au fur et à mesure du bétonnage, en veillant à ce que l'extrémité inférieure reste toujours suffisamment noyée dans le béton pour empêcher toute remontée d'eau dans celui-ci [2].

\section{Identification des matériaux pour béton coulé sous l'eau :}

\section{* Sable}

Dans cette recherche expérimentale, le sable $0 / 2$ choisi est un matériau lavé et criblé. Sa masse volumique absolue est de l'ordre de $2540 \mathrm{~kg} / \mathrm{m}^{3}$. Elle est comprise entre $2000 \mathrm{~kg} / \mathrm{m}^{3}$ et $3000 \mathrm{~kg} / \mathrm{m}^{3}$. Donc, ce sable est dit courant conformément à la norme NF $\mathrm{P}$ 18-554. Le tableau 1, représentant les spécifications de sable pour les bétons hydrauliques (NF P 18-541), montre que ce sable est conforme aux valeurs spécifiées [3].

Tableau 1. Caractéristiques du sable $0 / 2$

\begin{tabular}{|c|c|c|c|c|c|c|c|}
\hline \multicolumn{3}{|c|}{ Paramètres de position } & \multicolumn{4}{|c|}{ Paramètres de forme } & $\begin{array}{c}\text { Module } \\
\text { de } \\
\text { finesse }\end{array}$ \\
\hline$M_{d}$ & $D_{95}$ & $\%<\dot{a} 0.08$ & $M(m m)$ & $C u$ & $C c$ & $\sigma$ & $M F$ \\
\hline 0.37 & 1.25 & 1 & 0.27 & 2.56 & 1.11 & 0.22 & 1.73 \\
\hline
\end{tabular}

\section{* Gravillons}

Les gravillons $4 / 15$ proviennent de la carrière d'El Houareb existant dans le gouvernorat de Kairouan. Le tableau 2 récapitule les caractéristiques du gravier 4/15.

Tableau 2. Caractéristiques du gravier $4 / 15$

\begin{tabular}{|c|c|c|l|l|l|}
\hline \multicolumn{4}{|c|}{$\begin{array}{c}\text { Paramètres } \\
\text { de position }\end{array}$} & \multicolumn{5}{|c|}{ Paramètres de forme } \\
\hline$M_{d}$ & $D_{95}$ & $M(\mathrm{~mm})$ & $C_{U}$ & $C_{c}$ & $\sigma$ \\
\hline 10 & 18 & 7.91 & 1.88 & 0.95 & 1.81 \\
\hline
\end{tabular}

La masse volumique apparente $\left(\boldsymbol{\sigma}_{\text {app }}\right)$ vaut 1550 $\mathrm{kg} / \mathrm{m}^{3}$. La masse volumique absolue $\left(\sigma_{g}\right)$ est égale à 2730 $\mathrm{kg} / \mathrm{m}^{3}$. Elle est comprise entre $2000 \mathrm{~kg} / \mathrm{m}^{3}$ et $3000 \mathrm{~kg} / \mathrm{m}^{3}$.

\section{* Ciments : Ciment portland I-32.5. HRS}

Dans cette étude expérimentale, on a utilisé un ciment portland I-32.5. HRS à haute résistance chimique aux sulfates. Il est conforme aux normes tunisienne NT 47.01 et NT 47.26. Le tableau 3 récapitule les caractéristiques de ce ciment.

Tableau 3. Caractéristiques du ciment I-32.5.HRS

\begin{tabular}{|c|c|c|c|c|}
\hline $\begin{array}{c}\text { Type } \\
\text { de } \\
\text { ciment }\end{array}$ & \multicolumn{2}{|c|}{$\begin{array}{c}\text { Propriétés } \\
\text { chimiques }\end{array}$} & \multicolumn{2}{|c|}{ Propriétés mécaniques } \\
\hline \multirow{2}{\text{I-}}{} & $\%$ & $<$ & \multicolumn{2}{|c|}{ Résistance à la compression } \\
\cline { 3 - 5 } 32.5. & $\mathrm{SO}_{3}$ & $3,5 \%$ & $\mathrm{R}_{\mathrm{c}}$ à $28 \mathrm{j}$ & $\begin{array}{c}\mathrm{R}_{\mathrm{c}} \text { à } 7 \mathrm{j} \\
\text { minimale }\end{array}$ \\
\hline
\end{tabular}

\begin{tabular}{|l|l|l|c|c|}
\hline \multirow{2}{*}{} & \multirow{2}{*}{} & $\begin{array}{c}\text { minimale } \\
(\mathrm{MPa})\end{array}$ & $(\mathrm{MPa})$ \\
& & 32.5 & 16 \\
\hline
\end{tabular}

\section{* Eau de gâchage (la norme NF P18-303)}

L'eau de gâchage utilisée dans cette étude est l'eau distribuée dans le réseau d'eau potable.

\section{* Adjuvants}

-Adjuvant colloïdal (AWA): Pour augmenter la résistance au lavage du béton, on a utilisé le (AWA) destiné à la fabrication des bétons et mortiers coulés sous l'eau. L'ajout de (AWA) dans un béton confère à celui-ci un effet thixotropique (collant) [4]. Le tableau 4 récapitule les caractéristiques du (AWA).

Tableau 4. Caractéristiques du (AWA)

\begin{tabular}{|c|c|c|c|c|}
\hline $\begin{array}{c}\text { Densité } \\
\text { apparente }\end{array}$ & Nature & Couleur & $\begin{array}{c}\text { Domaines } \\
\text { d'utilisation }\end{array}$ & $\begin{array}{c}\text { Plage } \\
\text { d'utilisation }\end{array}$ \\
\hline 0.5 & poudre & grise & $\begin{array}{c}\text { Tout } \\
\text { bétonnage } \\
\text { en présence }\end{array}$ & $\begin{array}{c}\text { là3 doses de } \\
\text { d'eau de }\end{array}$ \\
& & & béton \\
\hline
\end{tabular}

-Un super plastifiant $(S P)$ : (SP) est un super plastifiant haut réducteur d'eau [5]. Il est d'aspect brun clair. Le (SP) est ajouté, soit en même temps que l'eau de gâchage soit en différé dans le béton préalablement mouillé avec une fraction de l'eau de gâchage [6]. Le tableau 5 récapitule les caractéristiques du (SP). L'examen de la figure 1, illustrant le temps d'écoulement en fonction d'A/C, permet de distinguer deux parties. La première partie, variant de $0 \%$ à $1.6 \%$; elle est décroissante et donne un temps d'écoulement minimal de l'ordre de $12.60 \mathrm{~s}$. Pour des valeurs de A/C plus importantes allant jusqu' à $2 \%$ la courbe devient pratiquement constante. Il résulte que le $(\mathrm{SP})$ possède un optimum de fluidité donné par un rapport $(\mathrm{A} / \mathrm{C})$ de $1.6 \%$ et un temps d'écoulement $\left(\mathrm{t}_{\mathrm{c}}\right)$ de $12.60 \mathrm{~s}$.

Tableau 5. Caractéristiques du SP

\begin{tabular}{|c|c|c|c|c|}
\hline Densité & $\mathbf{p H}$ & $\begin{array}{c}\mathbf{N a}_{2} \mathbf{O} \\
(\%)\end{array}$ & $\begin{array}{c}\mathbf{C l}^{-} \\
(\%)\end{array}$ & $\begin{array}{c}\text { Extrait } \\
\mathbf{s e c}\end{array}$ \\
\hline 1.06 & 4 & $<1$ & $<0.1$ & 31.5 \\
\hline
\end{tabular}

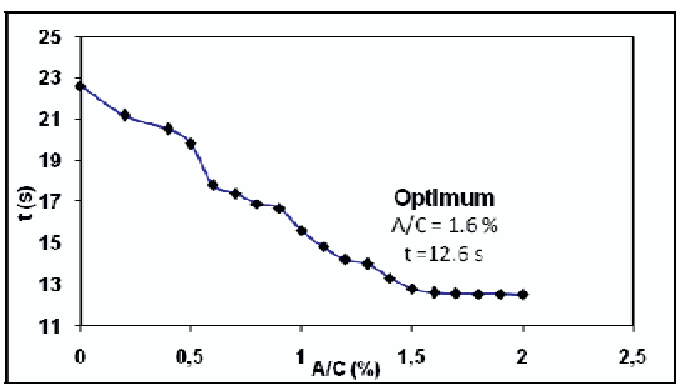

Fig. 1. Temps d'écoulement en fonction du rapport A/C du SP

\section{Programme expérimental}

\subsection{Conception du mélange}


D'après l'analyse de la courbe granulométrique du gravier $4 / 15$ on a donc $\mathrm{D}=16 \mathrm{~mm}$. Le coefficient $\mathrm{K}$ est égal à 10 tel que le volume de gravillons soit proche du volume de sable [7].

-Le point $\mathrm{O}$ est de coordonnées $(\mathrm{x}=0, \mathrm{y}=0)$

-Le point $\mathrm{B}$ est confondu avec le point d'ordonnée $100 \%$ de la courbe granulométrique du gravier 4/15.

-Le point $\mathrm{A}$ de brisure est de coordonnées en abscisse $\mathrm{X}=\mathrm{D} / 2$ et en ordonnée $\mathrm{y}=50-\mathrm{D}^{1 / 2}+\mathrm{k}$

Donc $\mathrm{x}=\frac{D}{2}=\frac{16}{2}=8 \mathrm{~mm}$ et $\mathrm{y}=50-\sqrt{16}+10=56[7]$.

Le point de brisure A est de coordonnées $(x=8, y=56)$

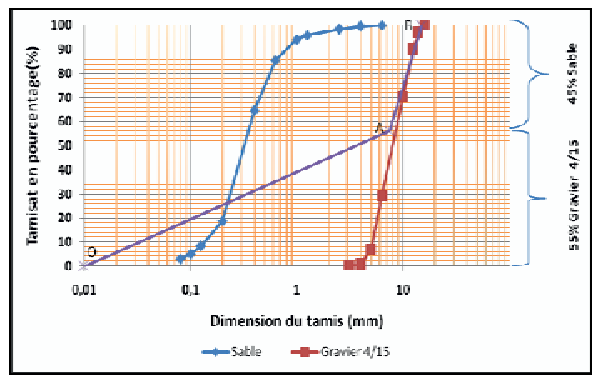

Fig.2. Optimisation du squelette granulaire du mélange sable et gravier $4 / 15$

\subsection{Détermination des pourcentages en granulats et la composition du béton coulé sous l'eau : "Méthode de Dreux Gorisse Modifiée »}

Pour la valeur de $\mathrm{k}=10$, l'application de la méthode conduit à un squelette granulaire composé de $55 \%$ de gravillons et de $45 \%$ de sable.

Le volume optimum des fines est

$$
\mathrm{V}_{\mathrm{FO}}=\frac{200}{\mathbf{F} \sqrt{D}}+1.3 k(1)
$$

Avec :

$\mathrm{D}$ : diamètre du plus gros granulat du squelette granulaire

$\mathrm{K}$ : le terme correctif définit ci-dessus

$$
\mathrm{V}_{\mathrm{FO}}=\frac{200}{5 \sqrt{16}}+1.3 k \times 10=\frac{200}{5 \sqrt{16}}+13=128 l / \mathrm{m}^{3}(2)
$$

$\mathrm{V}_{\mathrm{EP}}$ : le volume d'eau permettant de remplir la porosité du squelette solide [8]

$$
\begin{aligned}
& \mathrm{V}_{\mathrm{Ep}}=1.2 \times \mathrm{V}_{\mathrm{FO}}(\mathbf{3}) \\
& \mathrm{V}_{\mathrm{EP}}=154 \mathrm{l} / \mathrm{m}^{3} \text { (4) }
\end{aligned}
$$

Soit h l'affaissement au cône d'Abrâms visé en $\mathrm{cm}$

Pour atteindre cet affaissement il faut ajouter au volume $\mathrm{V}_{\mathrm{EP}}$ : un volume $\mathrm{V}_{\mathrm{ES}}$ mesuré en $1 / \mathrm{m}^{3}[\mathbf{8}]$.

$$
\mathrm{V}_{\mathrm{ES}}=20 \times \ln (\mathrm{h}+1)(\mathbf{5})
$$

Pour passer d'un affaissement de $25 \mathrm{~cm}$ à un étalement de l'ordre de $60 \mathrm{~cm}$, on considère qu'il faut rajouter un volume d'eau d'environ $10 \mathrm{l} / \mathrm{m}^{3}$ [7].

$$
\mathrm{V}_{\mathrm{ES}}=20 \times \ln (\mathrm{h}+1)+10=75 \mathrm{l} / \mathrm{m}^{3} \mathbf{( 6 )}
$$

Le volume total d'eau en $1 / \mathrm{m}^{3}$ est

$$
\begin{aligned}
& V_{E}=V_{E S}+V_{E P} \text { (7) } \\
& V_{E}=2291 / m^{3} \text { (8) }
\end{aligned}
$$

Le volume des vides en $1 / \mathrm{m}^{3}$ est

$$
\begin{aligned}
& \mathrm{V}_{\mathrm{V}}=\frac{\mathrm{VE}}{2 h}(9) \\
& \mathrm{V}_{\mathrm{V}}=\frac{\mathrm{VE}}{2 \bar{h}}=\frac{229}{2 \times 25}=51 / \mathrm{m}^{3}
\end{aligned}
$$

Le volume de la pâte

$$
\mathrm{V}_{\text {pâte }}=\mathrm{V}_{\mathrm{V}}+\mathrm{V}_{\mathrm{E}}+\mathrm{V}_{\mathrm{FO}}=362 \mathrm{l} / \mathrm{m}^{3} \text { (11) }
$$

La compacité du mélange est

$$
c=(1-\mathrm{p})=\frac{V_{\mathrm{c}}}{V_{c}+V_{\mathrm{E}}+V_{\nu}}=\frac{128}{362}=0.35
$$

Avec

$\mathrm{P}$ : porosité du mélange

La résistance à la compression à 28jours du béton optimisé de base est

$$
\mathrm{f}_{\mathrm{cm} 28}^{\prime}=\mathrm{G} \times \sigma_{\mathrm{c} 28}(1-\mathrm{P})^{2}=\mathrm{G} \times \sigma_{\mathrm{c} 28} \times \mathrm{c}^{2} \text { (13) }
$$

Avec

G: un coefficient sans dimension appelé coefficient granulaire qui dépend de la nature des granulats. Lorsque $\mathrm{G}$ n'est pas connu, on prend $\mathrm{G}=5$.

C : compacité de la pâte

$\sigma_{\mathrm{c} 28}:$ classe vraie du ciment

$$
\mathrm{f}^{\prime}{ }_{\mathrm{cm} 28}=5 \times 42.5 \times(0.35)^{2}=26 \mathrm{Mpa}<35 \mathrm{Mpa} \text { (14) }
$$

La résistance à la compression à 28 jours du béton optimisé de base est inférieure à celle qu'on veut atteindre. Donc on doit augmenter cette résistance en réduisant le dosage en eau par l'introduction d'un super plastifiant.

La réduction d'eau est :

$$
\begin{gathered}
\Delta \mathrm{V}_{\mathrm{E}}=\mathrm{V}_{\text {pâte }} \times \sqrt{\frac{\text { fanng }}{G \times \sigma e z 8}}-V F O \text { (15) } \\
\Delta \mathrm{V}_{\mathrm{E}}=362 \times \sqrt{\frac{35}{5 \times 42.5}}-128 \text { (16) } \\
\Delta \mathrm{V}_{\mathrm{E}}=191 / \mathrm{m}^{3}(\mathbf{1 7})
\end{gathered}
$$

$\mathrm{f}_{\mathrm{cm} 28}$ : la résistance moyenne à la compression qu'on veut atteindre à 28 jours en $\mathrm{MPa}$. Donc le volume des fines (ciment) est :

$$
\mathrm{V}_{\mathrm{c}}=\mathrm{V}_{\mathrm{FO}}+\Delta \mathrm{V}_{\mathrm{E}}=147 \mathrm{l} / \mathrm{m}^{3} \text { (18) }
$$

Après la réduction, le volume $\mathrm{d}$ 'eau est

$$
\mathrm{V}_{\mathrm{E}}=229-19=210 \mathrm{l} / \mathrm{m}^{3} \text { (19) }
$$

La masse de ciment est

$$
\mathrm{m}_{\mathrm{c}}=\mathrm{V}_{\mathrm{c}} \times \sigma_{\mathrm{c}}=147 \times 3.15=463 \mathrm{~kg} / \mathrm{m}^{3}
$$

$\sigma_{\mathrm{c}}$ : la masse volumique du ciment

$\mathrm{V}_{\mathrm{c}}$ : volume du ciment en $1 / \mathrm{m}^{3}$

Or d'après l'optimisation du (SP), le rapport massique est $\mathrm{A} / \mathrm{C}=1.6 \%$. Avec :

A : masse de super plastifiant

$\mathrm{C}$ : masse du ciment

Donc la masse du super plastifiant est $\mathrm{A}=\mathrm{C} \times 0.016=463 \times 0.016=7.4 \mathrm{~kg} / \mathrm{m}^{3}(\mathbf{2 1})$

Or la masse volumique du super plastifiant est $\sigma_{\mathrm{A}}=1.06 \mathrm{~kg} / 1 \mathrm{donc}$ son volume est

$$
V A=\frac{A}{\sigma A}=\frac{7.4}{1.06}
$$

$$
\mathrm{V}_{\mathrm{A}=6.98 \approx 71 / \mathrm{m}^{3}(\mathbf{2 3})}
$$

D'où le volume total de la pâte est

$$
\begin{gathered}
\mathrm{V}_{\text {pâte }}=\mathrm{V}_{\mathrm{V}}+\mathrm{V}_{\mathrm{E}}+\mathrm{V}_{\mathrm{c}}+\mathrm{V}_{\mathrm{A}} \text { (24) } \\
\mathrm{V}_{\text {pâte }}=3691 / \mathrm{m}^{3} \text { (25) }
\end{gathered}
$$

$\mathrm{V}_{\mathrm{A}}=$ volume du super plastifiant en $1 / \mathrm{m}^{3}$

Pour un mètre cube de béton, le volume des granulats est

$$
\mathrm{V}_{\text {granulats }}=1000-\mathrm{V}_{\text {pâte }} \text { (26) }
$$




$$
\mathrm{V}_{\text {granulats }}=1000-369=631 \mathrm{l} / \mathrm{m}^{3}(27)
$$

L'application de la méthode de Dreux Gorisse conduit à un squelette granulaire composée de 55\% de gravillons et de $45 \%$ sable. D'où le volume du sable est

$$
\mathrm{Vs}=631 \times 0.45=284 \mathrm{l} / \mathrm{m}^{3}(\mathbf{2 8})
$$

D'où le volume du gravier est

$$
\mathrm{V}_{\mathrm{g}}=631 \times 0.55=347 \mathrm{l} / \mathrm{m}^{3} \text { (29) }
$$

La masse volumique du sable $0 / 2$ est $\sigma_{\mathrm{s}}=2.54 \mathrm{~kg} / \mathrm{m}^{3}(\mathbf{3 0})$

D'où la masse du sable est $\mathrm{m}_{\mathrm{s}}=\sigma \mathrm{s} \times \mathrm{Vs}_{\mathrm{s}}$ (31)

$$
\mathrm{m}_{\mathrm{s}}=2.54 \times 284=721 \mathrm{~kg} / \mathrm{m}^{3} \mathbf{( 3 2 )}
$$

La masse volumique du gravier est : $\sigma_{\mathrm{g}}=2.73 \mathrm{~kg} / \mathrm{m}^{3}(\mathbf{3 3})$

D'où la masse du gravier est $\mathrm{m}_{\mathrm{g}}=\sigma_{\mathrm{g}} \times \mathrm{V}_{\mathrm{g}}$ (34)

$$
\mathrm{m}_{\mathrm{g}}=2.73 \times 347=948 \mathrm{~kg} / \mathrm{m}^{3} \text { (35) }
$$

Le dosage pour $1 \mathrm{~m}^{3}$ est récapitulé dans le tableau 6 .

Tableau 6. Dosage retenu pour un mètre cube de béton

\begin{tabular}{|c|c|c|c|c|c|c|}
\hline & C & G & S & E & A & AIR \\
\hline m(kg) & 463 & 948 & 721 & 210 & 7.4 & \\
\hline V(l) & 147 & 284 & 347 & 210 & 7 & 5 \\
\hline
\end{tabular}

\section{4 .3.Optimisation de l'adjuvant colloïdal (AWA)}

L'optimisation de l'adjuvant d'anti lavage (AWA) pour le béton coulé sous l'eau se fait par le test au plongeon selon la norme (CRD C61). En effet le dosage de l'adjuvant est varié de $0 \%$ à $6 \%$ en pourcentage massique du ciment [6].L'examen de la figure 3, illustrant la perte de masse temps d'écoulement en fonction d'A/C, permet de distinguer deux parties. On distingue deux intervalles. Dans le premier intervalle, AWA varie de 0 à $3 \%$; la courbe est décroissante avec une perte de masse minimale de l'ordre de $13.64 \%$. Pour des dosages plus importants allant jusqu'à $6 \%$ la courbe devient presque constante. Il résulte que l'adjuvant colloïdal (AWA) possède un optimum de résistances au lavage donné par un dosage de $3 \%$ et une perte de masse minimale de $13.64 \%$.

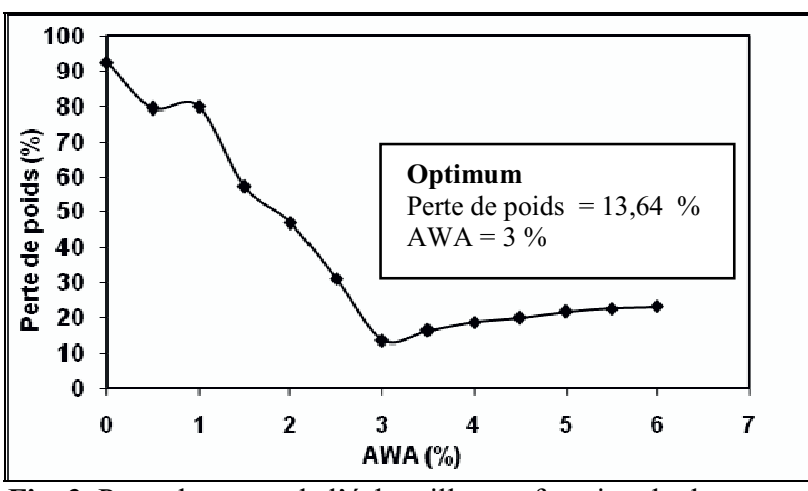

Fig .3. Perte de masse de l'échantillon en fonction du dosage en adjuvant colloïdal

Les résultats de la formulation par la méthode de «Dreux Gorisse modifiée » sont validés puisqu'ils respectent les exigences de la norme NF EN 206-1[7], voir tableau 7.

Tableau 7.Vérification de la formulation du béton

\begin{tabular}{|c|c|c|c|}
\hline Mélange & $\mathrm{E}_{\text {eff }^{\prime} \text { liant }}$ & $\begin{array}{c}\text { Teneur } \\
\text { minimale } \\
\text { en liant } \\
\text { équivalent } \\
\left(\mathrm{kg} / \mathrm{m}^{3}\right)\end{array}$ & $\begin{array}{c}\text { Résistance } \\
\text { caractéristique } \\
\text { minimale } \\
(\mathrm{MPa})\end{array}$ \\
\hline béton & $\mathbf{0 . 4 5} \leq 0.55$ & $\mathbf{4 6 3} \geq 330$ & $\mathbf{3 0}$ \\
\hline
\end{tabular}

\section{Résultats expérimentaux et discussion}

\subsection{Propriétés physiques à l'état frais du béton coulé sous l'eau :}

Dans le but d'étudier l'influence de l'ajout de l'adjuvant colloïdal (AWA) sur les propriétés des bétons frais, on a réalisé deux mélanges. Le tableau 8 présente les compositions du mélange 1 et du mélange 2 . Le mélange 1 ne contient pas l'adjuvant (AWA) alors que le mélange 2 contient l'adjuvant (AWA).

Tableau 8 .Composition des mélanges d'étude

\begin{tabular}{|c|c|c|c|c|c|c|c|c|c|}
\hline Mélange & C & G & S & E & A & AIR & AWA & G/S & E/C \\
\hline $\mathbf{1}$ & 463 & 948 & 721 & 210 & 7 & 5 & 0 & 1.3 & 0.45 \\
\hline $\mathbf{2}$ & 463 & 948 & 721 & 210 & 7 & 5 & 13.89 & 1.3 & 0.45 \\
\hline
\end{tabular}

Les résultats des tests d'étalement au cône d'Abrâms, de stabilité au tamis et de résistance au lavage sont présentés dans le tableau 9.

Tableau 9 .Caractérisation des mélanges d'étude

\begin{tabular}{|l|c|c|}
\hline Mélange & $\mathbf{1}$ & $\mathbf{2}$ \\
\hline $\begin{array}{l}\text { Diamètre } \\
\text { d'étalement } \\
\text { D(mm) }\end{array}$ & 640 & 600 \\
\hline $\begin{array}{l}\text { Temps } \\
\text { d'écoulement } \\
\text { T500 (s) }\end{array}$ & 2 & 3.15 \\
\hline $\begin{array}{l}\text { Perte de } \\
\text { masse } \\
\text { (\%) }\end{array}$ & 92.44 & 13.64 \\
\hline $\begin{array}{l}\text { Stabilité au } \\
\text { tamis }\end{array}$ & 13 & 7 \\
\hline
\end{tabular}




\begin{tabular}{|l|l|l|}
\hline J(\%) & & \\
\hline
\end{tabular}

\subsubsection{Conception des mélanges :}

Pour le mélange 1, le béton est fluide, il souffre du phénomène de ségrégation dynamique et sa résistance au lavage de l'eau de mer est très faible. Le mélange 2 est donc plus visqueux que le mélange 1 puisque son temps d'écoulement $T_{\mathbf{5 0 0}}$ est plus important que celui du mélange 1 .

\subsubsection{Influence de l'adjuvant d'anti lavage}

En faisant la comparaison entre le comportement des deux mélanges à l'état frais; on peut conclure que l'ajout de l'adjuvant colloïdal a pour effet :

-D'augmenter la viscosité du béton ce qui a entrainé la réduction de l'étalement au cône d'Abrâms de $640 \mathrm{~mm}$ à $600 \mathrm{~mm}$, c'est-à-dire que l'adjuvant colloïdal a diminué l'écoulement du béton

-D'augmenter la stabilité au tamis puisque la stabilité du mélange 1 est égale à13\% alors que celle du mélange 2 , comportant l'adjuvant d'anti lavage (AWA), est égale à $7 \%$.

-De diminuer la perte de masse du béton résultant du lavage de $92.44 \%$ à $13.64 \%$, l'adjuvant colloïdal (AWA) permet donc de diminuer le délavage des bétons coulés sous l'eau.

\subsection{Propriétés physiques à l'état durci du béton coulé sous l'eau :}

\subsubsection{Résistance à la compression}

- A 28 jours: Afin de calculer la résistance à la compression du béton coulé sous l'eau, nous avons confectionné 3 éprouvettes cylindriques de dimension $16 \times 32$. Ces éprouvettes sont conservées dans l'eau pendant 28 jours. La résistance à la compression est déterminée par l'essai d'écrasement. Les résultats de l'écrasement des éprouvettes sont rapportés dans le tableau 10.

Tableau 10. Composition des éprouvettes confectionnées

\begin{tabular}{|c|c|c|c|}
\hline & $\begin{array}{c}\text { Eprouvette } \\
1\end{array}$ & $\begin{array}{c}\text { Eprouvette } \\
2\end{array}$ & $\begin{array}{c}\text { Eprouvette } \\
3\end{array}$ \\
\hline $\begin{array}{c}\text { Résistance à } \\
\text { la } \\
\text { compression } \\
\text { fc }_{28}(\mathrm{MPa})\end{array}$ & 34.25 & 33 & 36.4 \\
\hline
\end{tabular}

-A 90 jours: Pour vérifier la compatibilité chimique entre le super plastifiant, l'adjuvant colloïdal et le ciment, trois éprouvettes cylindriques de dimension $16 \times 32$ sont confectionnées. Après 90 jours de conservation dans l'eau, la résistance à la compression est déterminée par l'essai d'écrasement. Les résultats des essais de compression sont rapportés dans le tableau 11.

Tableau 11. Composition des éprouvettes confectionnées

\begin{tabular}{|c|c|c|c|}
\hline & $\begin{array}{c}\text { Eprouvette } \\
4\end{array}$ & $\begin{array}{c}\text { Eprouvette } \\
5\end{array}$ & $\begin{array}{c}\text { Eprouvette } \\
6\end{array}$ \\
\hline $\begin{array}{c}\text { Résistance à } \\
\text { la } \\
\text { compression } \\
\text { fc90(MPa) }\end{array}$ & 36.74 & 38.23 & 35.8 \\
\hline
\end{tabular}

On constate que les trois résultats sont supérieurs à la force caractéristique, $\mathrm{f}_{\mathbf{c k 2 8}}=30 \mathrm{MPa}$

La force moyenne $\mathrm{f}_{\mathbf{c m} 90}=36.92 \approx 37 \mathrm{MPa}>\mathrm{fcm}_{\mathrm{cm}} 28=35 \mathrm{MPa}$ Donc la résistance à la compression du béton est acceptable. On remarque une augmentation légère de la résistance à la compression à 90 jours par rapport à celle à 28 jours. Donc les deux adjuvants et le ciment utilisés dans la conception du mélange sont compatibles.

\subsubsection{Vitesse des ultrasons}

La vitesse des ultrasons a été mesurée entre transmission directe le long de l'éprouvette. Pour évaluer cette vitesse, on a confectionné des éprouvettes 1, 2 et 3 à partir du béton coulé sous l'eau (mélange 2) et l'éprouvette 7 à partir du béton du mélange 1 . Ces éprouvettes de dimension $16 \times 32$ sont conservées dans l'eau pendant 28 jours. Les résultats des essais expérimentaux sont assemblés dans le tableau 12.

\section{a) Pour 28 jours}

Tableau 12. Valeurs de la vitesse des ultrasons à28 jours

\begin{tabular}{|c|c|c|c|c|}
\hline & $\begin{array}{c}\text { Eprouvette } \\
1\end{array}$ & $\begin{array}{c}\text { Eprouvette } \\
2\end{array}$ & $\begin{array}{c}\text { Eprouvette } \\
3\end{array}$ & $\begin{array}{c}\text { Eprouvette } \\
7\end{array}$ \\
\hline $\begin{array}{c}\text { Vitesse } \\
\text { des } \\
\text { ultrasons } \\
\mathbf{V}_{28}(\mathrm{~m} / \mathbf{s})\end{array}$ & 4260 & 4150 & 4285 & 4100 \\
\hline
\end{tabular}

On remarque que les valeurs des vitesses ultrasoniques $\mathrm{du}$ béton $\mathrm{du}$ mélange $\mathbf{2}$ sont plus importantes que celle du béton du mélange 1. Ceci est expliqué par l'homogénéité et la densité du béton coulé sous l'eau (mélange 2) qui sont plus intéressantes que celles du béton ne comportant pas l'adjuvant d'anti lavage (mélange 1). L'adjuvant d'anti lavage (AWA) confère au béton durci une structure cristalline plus dense et plus compacte.

\section{b) Pour 90 jours}

Pour évaluer la vitesse des ultrasons à 90 jours, on a confectionné les éprouvettes 4,5 et 6 à partir du béton coulé sous l'eau (mélange 2) et l'éprouvette 8 à partir du béton du mélange 1. Ces éprouvettes de dimension $16 \times 32$ sont conservées dans l'eau pendant 90 jours. Les résultats des essais expérimentaux sont assemblés dans le tableau 13.

Tableau 13. Valeurs de la vitesse des ultrasons à90 jours

\begin{tabular}{|c|c|c|c|c|}
\hline & $\begin{array}{c}\text { Eprouvette } \\
\mathbf{4}\end{array}$ & $\begin{array}{c}\text { Eprouvette } \\
\mathbf{5}\end{array}$ & $\begin{array}{c}\text { Eprouvette } \\
\mathbf{6}\end{array}$ & $\begin{array}{c}\text { Eprouvette } \\
8\end{array}$ \\
\hline $\begin{array}{c}\text { Vitesse } \\
\text { des }\end{array}$ & 4340 & 4390 & 4310 & 4200 \\
$\begin{array}{c}\text { ultrasons } \\
V_{90}(\mathrm{~m} / \mathrm{s})\end{array}$ & & & & \\
\hline
\end{tabular}

On remarque que les valeurs des vitesses ultrasoniques du béton $\mathrm{du}$ mélange 2 sont plus importantes que celle du béton du mélange 1 . Ceci peut être expliqué par l'homogénéité et la densité intéressante du béton coulé sous l'eau. En effet l'introduction du super plastifiant et de l'adjuvant colloïdal n'a pas d'effet négatif sur la qualité du béton. On peut dire que les adjuvants et le ciment utilisés dans la conception du béton coulé sous l'eau sont compatibles. 


\section{Conclusion}

La conception de la formulation des bétons coulés sous l'eau et le contrôle de leurs propriétés exigent une grande attention. Les caractéristiques des bétons coulés sous l'eau: la fluidité, la résistance au lavage et la ségrégation, l'homogénéité, la résistance du béton à la compression, la compatibilité chimique des adjuvants utilisés dans la formulation du béton et la durabilité sont dépendants de l'ajout des adjuvants au béton de base. La formulation du béton coulé sous l'eau par la méthode de Dreux Gorisse Modifiée mous a permis de donner la composition adéquate de béton. Cette formulation a été utilisée pour confectionner des pieux qui ont montré des résistances variables selon la teneur en adjuvants. Cependant cette étude nous a permis de développer un béton spécial grâce aux \% optimaux en adjuvants. Ce béton est susceptible d'être utilisé lorsqu'on désire obtenir certaine propriétés particulières précises et indispensable pour certaines applications.

\section{References}

1. Centre d'information sur le ciment et ses applications T93, Les bétons et les ouvrages en site maritime, 120 (2011).

2. J. Baron, R. Sauterey, Le béton hydraulique (1982).

3. NFP 18-554, NFP 18-555), Les granulats (2011).

4. Notice technique Sika, Edition Septembre 2003, Numéro 1.06, Version n ${ }^{\circ} 127$ (2004).

5. Notice technique Sika, Edition Décembre 2008, Numéro 2.04 y, Version nº 49 (2008).

6. C. Aticin, C. Jolicoeur and J.G. M. Acgregor, A look at certain characteristics of superplasticizers and their use the industry, (Concrete international, 16, No. 15, pp.45-52, 1994).

7. R. Lanchon, R. Dupain et S .Arroman, Granulats, sols, ciments et bétons, pp 161-169 (1994). 